\title{
Spot Detection in Images with Noisy Background
}

\author{
Denis Ferraretti ${ }^{1}$, Luca Casarotti ${ }^{1}$, Giacomo Gamberoni ${ }^{2}$, and Evelina Lamma ${ }^{1}$ \\ 1 ENDIF-Dipartimento di Ingegneria, Università di Ferrara, Ferrara, Italy \\ \{denis.ferraretti, evelina.lamma\}@unife.it, \\ luca.casarotti@student.unife.it \\ 2 intelliWARE snc, Ferrara, Italy \\ giacomo@i-ware.it
}

\begin{abstract}
One of the most recurrent problem in digital image processing applications is segmentation. Segmentation is the separation of components in the image: the ability to identify and to separate objects from the background. Depending on the application, this activity can be very difficult and segmentation accuracy is crucial in order to obtain reliable results. In this paper we propose an approach for spot detection in images with noisy background. The overall approach can be divided in three main steps: image segmentation, region labeling and selection. Three segmentation algorithms, based on global or local thresholding technique, are developed and tested in a real-world petroleum geology industrial application. To assess algorithm accuracy we use a simple voting technique: by a visual comparison of the results, three domain experts vote for the best algorithms. Results are encouraging, in terms of accuracy and time reduction, especially for the algorithm based on local thresholding technique.
\end{abstract}

Keywords: image segmentation, local thresholding, spot detection, petroleum geology application.

\section{Introduction}

In digital image processing a common task is partitioning an image into multiple areas that collectively cover the entire image. Segmentation subdivides an image into its constituent regions or objects. The level of detail to which the subdivision is carried depends on the problem being solved. That is, segmentation should stop when the objects or regions of interest in an application have been detected [1].

The goal of segmentation is to simplify and/or change the representation of an image into something that is more meaningful and easier to analyse. More precisely, image segmentation is the process of assigning a label to every pixel in an image such that pixels with the same label share certain visual characteristics. Each of the pixels in a region are similar with respect to some characteristic or computed property, such as color, intensity, or texture. Adjacent regions are significantly different with respect to the same characteristics [2]. 
Segmentation of non trivial images is one of the most difficult tasks in image processing. Segmentation accuracy determines the eventual success or failure of computerized analysis procedures. For this reason, considerable care should be taken to improve the probability of accurate segmentation.

In this paper we propose and experiment three algorithms, based on global and local thresholding techniques, for spot detection in noisy images. Algorithms take as input an image and give as result all the informations (such as position, perimeter and color) about small round areas in contrast with the backgorund, according to some visual criteria. We test the algorithms in a petroleum geology application using digital images provided by a borehole logging tool called FM! 1 . An interpretation of these acquired images (image logs) is usually made, by the petroleum geologist, to locate and quantify potential depth zones containing oil and gas. In this context the segmentation accuracy in spot detection is very important in order to produce reliable evaluation of the studied reservoir.

To assess algorithms accuracy we use a simple voting technique: by the direct observation of the output of chosen images at different depths of the well, three domain experts choose the best algorithm.

The paper is organized as follows: segmentation basic concepts and related work are outlined in Section 3.1. Three developed algorithms for spot detection are introduced in Section 3. A detailed explanation of experimental results over different images is given in Section 4. Finally, section 5] concludes the paper.

\section{Segmentation Algorithms}

In image analysis, one of the most recurrent problem is the separation of components in the image: the ability to identify and to separate objects from the background. This activity is called image segmentation. Segmentation algorithms are based on one of two basic properties of intensity values: discontinuity and similarity. In the first category, the approach is to partition an image based on abrupt changes in intensity, such as edges (i.e. Canny edge detector [15]). The principal approaches in the second category are based on partitioning an image into regions that are similar according to a set of predefined criteria. Thresholding, region growing (i.e. [17]), and region splitting and merging are examples of methods in this category.

Other proposed recent approaches [3 are segmentation based on the mean shift procedure [4], multiresolution segmentation of low-depth-of-field images [5], a Bayesian-framework-based segmentation involving the Markov chain Monte Carlo technique 6, and an EM-algorithm-based segmentation using a Gaussian mixture model[7]. A sequential segmentation approach that starts with texture features and refines segmentation using color features is explored in 8 . An unsupervised approach for segmentation of images containing homogeneous color/texture regions has been proposed in 9 .

${ }^{1}$ FMI (Fullbore Formation MicroImager) is the name of the tool used to acquire image logs based on resistivity measures within the borehole. 
In our work we focus on segmentation obtained by threshold operations. Let $f(x, y)$ be the function that describes our image. The image consists of a white object on a dark background. The extraction of the object can be achieved by defining a threshold $T$ and then comparing each pixel value with it. If the pixel value exceeds the threshold, the pixel is classified as an object pixel, if the value is lower than the threshold, the pixel is classified as a background pixel. The result is typically a binary image, where object pixels are represented in white and background pixel are represented in black.

Thresholding is a well known and straightforward technique and can be defined as an operation that involves a test against a $T$ function, which has the following form: $T=T[x, y, p(x, y), f(x, y)]$ where $f(x, y)$ is the function that describes the gray-level intensity for each pixel in the image; $p(x, y)$ describes some local properties for each pixel in the image; $(x, y)$ represents the position of pixels in the image. Depending on $T$, there are different types of threshold.

Global Threshold. It's the simplest operation: the threshold value $T$ is computed once for the whole image, and the image is thresholded by comparing each pixel value with $T$, as described above. The result depends on the shape of the image histogram. Many techniques have been proposed for the automatic computation of the threshold value. Otsu's method 10], for example, produces the threshold value that minimizes the intra-classes variance, defined as the weighted sum of the variance of the classes. The class weight correspond to the probability that a pixel belongs to that class.

Local Threshold. A global value for $T$ may not be enough in order to obtain good results in segmentation: the local approach, instead, computes a different threshold value for each pixel in the image, based on local statistical features. A neighbourhood is defined for each pixel: in this neighbourhood some statistical parameters are calculated (i.e.: mean, variance and median), which are used to calculate the threshold value $T(x, y)$. Niblack's algorithm[11] is an example of this type of thresholding.

The simple global threshold method can only be successful if the separation between the two classes (object vs. background) is clear. In real images, this assumption is typically not true. The local threshold method attempts to solve this issue, because the threshold value is not fixed, but calculated for each pixel on the basis of the local image features.

\section{Methodology}

The overall approach of spot detection in noisy background images can be divided in three steps: segmentation, labeling and selection. Segmentation identifies a set of interesting regions that are eligible to spots. Labeling provides the regions connected components in order to then select only those that are actual objects. 


\subsection{Image Segmentation}

We developed three different segmentation algorithms starting from two main methods. Our algorithms are made by the combinations of modified versions of well known image processing techniques such as image smoothing and thresholding. The first method uses a particular convolution mask and a global thresholding technique. In order to remove noise and unnecessary details, the image is first smoothed with a median filter. The convolution of this image with a circular derivative mask provides a new image where round areas or circular structures, approximately of the same size of the mask, are highlighted. The new image is then thresholded, using two global threshold values: $T_{\text {low }}$ and $T_{\text {high }}$. All the $(x, y)$ pixel where $f(x, y)<=T_{\text {low }}$ or $f(x, y)>=T_{\text {high }}$ are considered object pixels, others are background pixels. Using two different threshold is possible to find two types of spots: dark spots in light background and vice versa. Generally we use a percentile value to define two thresholds: $T_{\text {low }}$ is the 20 th percentile and $T_{\text {high }}$ the 80 th. In order to remove isolated pixels a opening morphological operator [16] is then applied. This method lead to the implementation of two different algorithm. The difference between these two implementations is in how the convolution manage the image background. In some cases (see Section 4) images can have zones with non-relevant or missing information. Our first algorithm considers these zones as background pixels, conversely in the second algorithm these pixels are considered null values (zones with no image).

The second method uses the approach based on local threshold. The first step is the application of a low-pass filter to the image. The purpose of the filter is to reduce the noise in the image. Then, once defined the size of the neighbourhood, intensity mean $(\mu)$ and variance $(\sigma)$ are computed for each pixel. For the calculation of the threshold value, the Niblack's algorithm[1] is applied: $T(x, y)=\mu(x, y)+k \sigma(x, y)$ Mean and variance are calculated in the neighbourhood of each pixel. Here, we are assuming that the image contains white objects on dark background. 2

In practice, two new images are built, starting from the original: in the first image, the pixel value is replaced with the mean value in the neighbourhood. In the second image, the pixel value is replaced with the variance calculated in the neighbourhood. To apply the Niblack's algorithm to the pixel $(x, y)$ is sufficient to get the pixel value from the original image, and its mean and variance from the new images. The Niblack's algorithm is reinforced with an additional constraint, based on the absolute value of the variance. Variance is related to the image contrast. A small value corresponds to an area fairly uniform in the image. To avoid the detection of false positives, a pixel must belong to a non uniform area: this means that the variance is to assume a high enough value. Hence a threshold value is needed to compare the variance. First the variance image histogram is built, then the threshold is selected as the value corresponding to an arbitrary percentile (for example, the 20th percentile). The pixel for which the variance is

\footnotetext{
${ }^{2}$ The detection of dark objects on light background can be achieved by inverting the original image (doing this causes that dark pixels turn into light pixels and vice versa) and then applying the same algorithm.
} 
lower than this value are automatically classified as background pixel. Niblack's algorithm is applied only to pixels that pass this test.

In order to detect light and dark objects, the method is applied to the original image and to the inverted image. As before, the opening morphological operator is then applied to the binary images, in order to smooth the contours of the regions identified.

\subsection{Image Labeling and Region Selection}

The second step in the proposed approach is aimed at identifying and labeling the connected components resulting from the segmentation process. Once we obtain a binary image a labeling algorithm is applied to detect all the image regions. The labeling algorithm identifies the connected components in an image and assigns each component a unique label. The algorithm runs an image scan and groups its pixels into regions, based on pixel connectivity. This procedure is often applied to binary images, resulting from segmentation. Once complete, the procedure returns a list of connected regions that were found in the image. Each region should represent an image object.

Finally in the last step, for each identified region a test is applied on the size and shape. In particular, the tested parameters might be: area, roundness and ratio (ratio between maximum height and maximum width). These tests prevent the algorithm from detecting regions which do not correspond to actual objects.

\section{Experimental Results}

We test our approach in a real-world petroleum geology application: the porosity evaluation of a rock formation in oil and gas reservoir. Image logs are digital images acquired by a special logging tool (FMI tool) within a borehole. They represent resistivity measurements of the rock formation taken by the wellbore surface. Image logs supply fundamental information on the characteristics of the reservoir sections explored and hold important information on the structural, lithological, textural and petrophysical properties of the rocks. Resistivity measurements are converted into gray-level intensity values, and each measurement corresponds to a pixel in the FMI image. This image is the unrolled version of the well surface and it is made by six vertical strips of measurement. There is a strip for each pad of sensors in the FMI tool, see Figure 2(a) for an example.

To estimate the porosity of the rocks from the image, we are interested in the detection of roughly circular areas, in contrast with the background. These spots are called vugs or vacuoles (see Figure 1 for an example).

Three different algorithms were implemented: the first two (algorithm 1 and 2) are very similar, and use the approach based on convolution. The third (algorithm 3) is an implementation of the local threshold method described in 3.1. All the algorithms are written in JAVA. To determine which method is most suitable for this task, a test was performed on an entire well FMI image. The analysis is carried out through a sliding window technique. From the main image, 300 pixel height windows are extracted, and algorithms are applied directly 

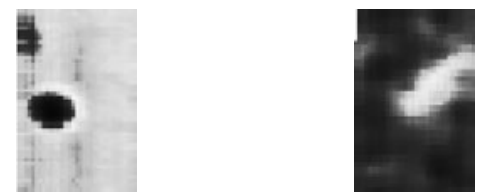

Fig. 1. Dark vug in a light background (on the left) and vice versa (on the right)

to them. Windows are partially overlapping: this is designed to improve the accuracy detection near the edges of the windows. Once completed the analysis on the entire well, in order to evaluate the results, about ten windows, considered significant, have been taken: windows, namely, showing the most common situations in which the geologist is interested. For example, a window containing a lot of small sized vugs was selected, rather than a window with a few large vacuoles. The chosen windows, and the three results for each of them, were shown to three geologists: it was asked them, for each window, to vote the algorithm (or the algorithms) that produced best results. At the end of the procedure, all votes were collected and a ranking was produced.

In our experiment algorithm 1 and 2 have a $7 \times 7$ pixel smoothing filter and a 9x9 pixel circular derivative convolution mask. Algorithm 3 runs with a $5 \times 5$ pixel smoothing filter; the radius of the neighbourhood is 13 pixel and $k=0.5$ in the Niblack's algorithm. Once each image region is labeled, a test is applied on the size and shape. In our work the total area of each region must be in the range 25 - 500 pixel. Roundness is defined as roundness $=\frac{4 \pi A}{p^{2}}$ where $A$ is the region area and $p$ is the perimeter. All the regions with a roundness lower than 0.25 pass the test and can be considered as vugs. The last test is based on the width-height ratio: for each region the maximum width and height are computed and only if the ratios width/height and height/width are greater than 1.8, the region pass the test. Details on the vote are shown in Table[1.

Table 1. Each geologist votes for the best algorithms (algorithm 1,2 or 3) for each well depth. Cells contains geologist choice.

\begin{tabular}{|l|c|c|c|}
\hline & Geologist A & Geologist B & Geologist C \\
\hline depth1 & 1,3 & 2,3 & 1 \\
depth2 & 2,3 & 2 & 3 \\
depth3 & 2,3 & 2 & 3 \\
depth4 & 3 & 3 & 3 \\
depth5 & 2 & $n . d$. & 3 \\
depth6 & 3 & 3 & 2 \\
depth 7 & 2,3 & 2 & 3 \\
depth8 & 2 & 2 & 3 \\
depth9 & 3 & $n . d$. & 3 \\
\hline
\end{tabular}




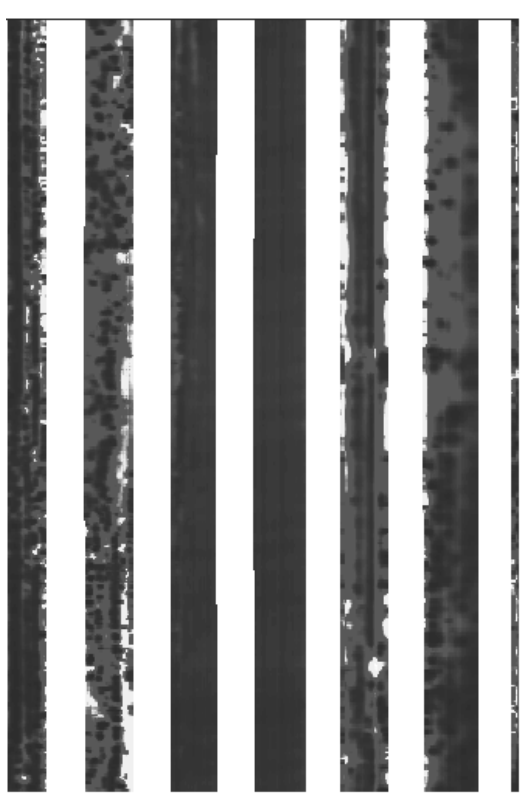

(a) Input image.

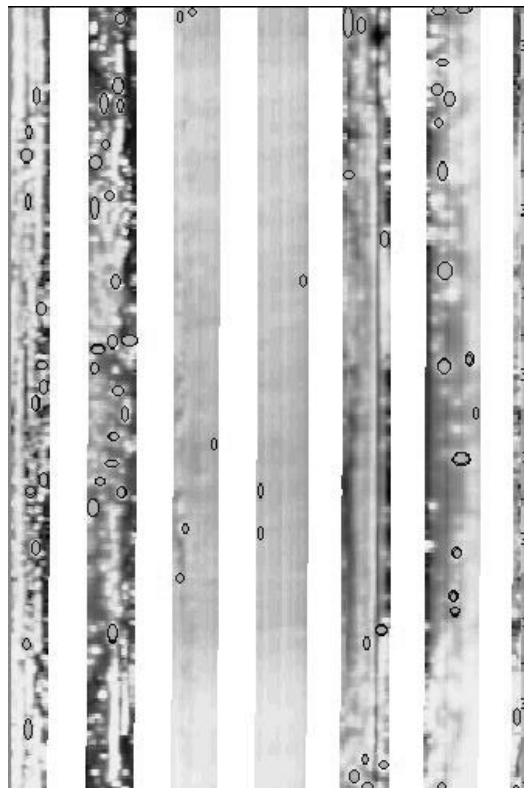

(c) Algorithm 2 detected spots.

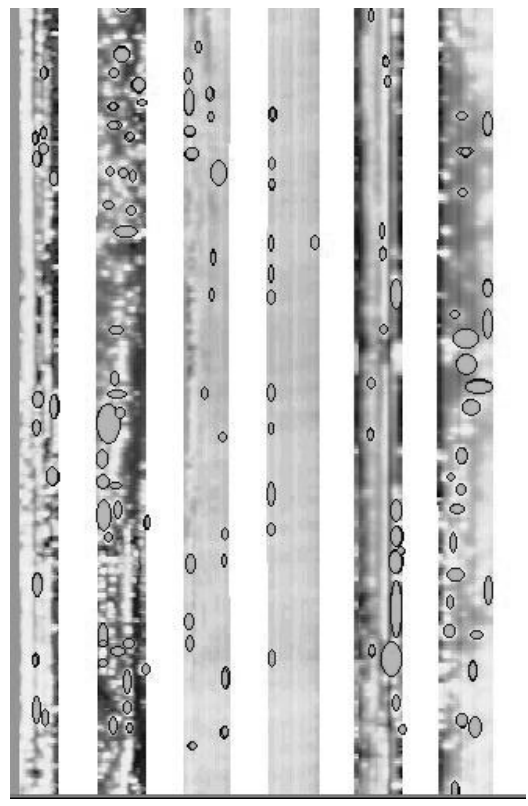

(b) Algorithm 1 detected spots.

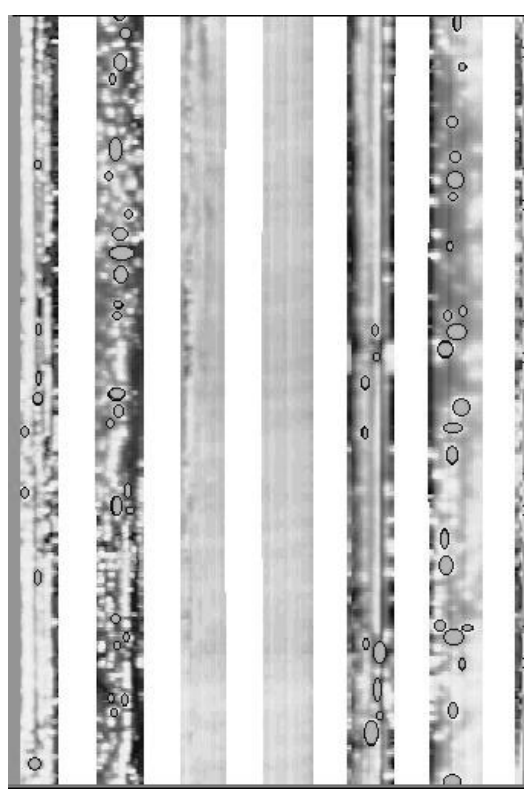

(d) Algorithm 3 detected spots.

Fig. 2. Example of gray-level image input (a) and output (b,c,d) at depth1. In output images, detected vugs are round grey area with black thin border. 


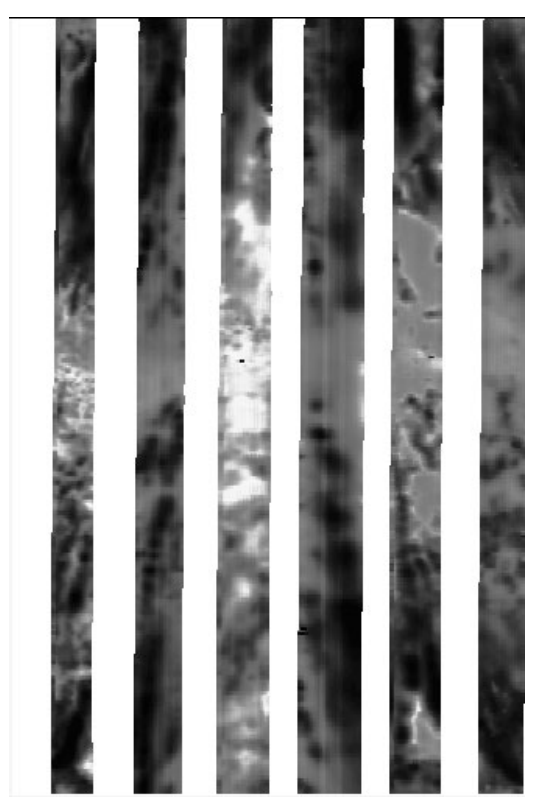

(a) Input image.

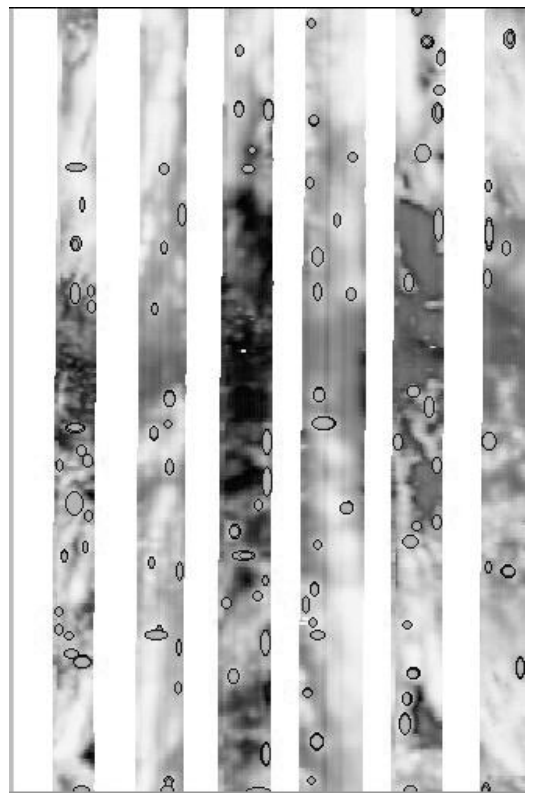

(c) Algorithm 2 detected spots.

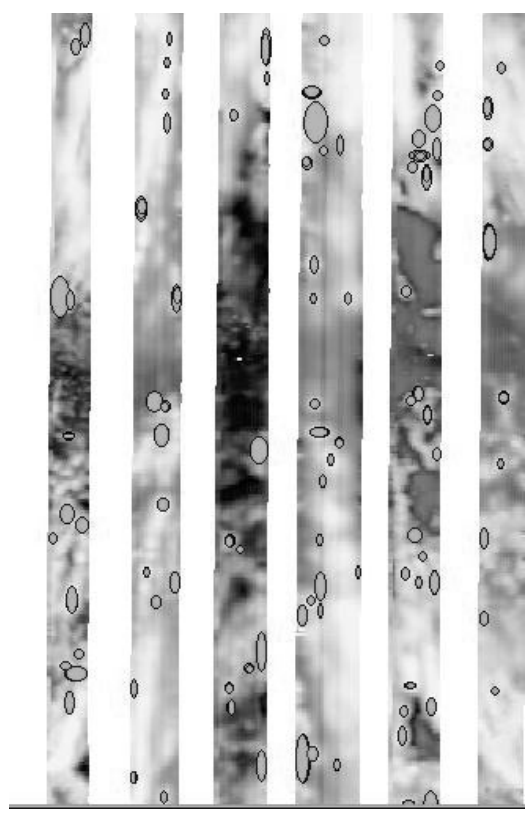

(b) Algorithm 1 detected spots.

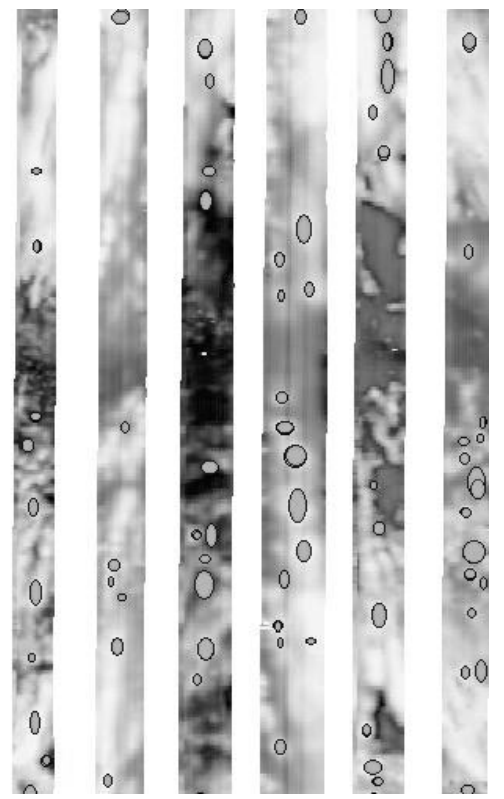

(d) Algorithm 3 detected spots.

Fig. 3. Example of gray-level image input (a) and output (b,c,d) at depth2. In output images, detected vugs are round grey area with black thin border. 
Final ranking is algorithm 1 has 2 votes, algorithm 2 has 11 votes and algorithm 3 receives 17 votes.

In Figure 2 the input image (depth1) shows a lots of small vugs, with a low contrast with respect to the background; two strips in the middle are very dark due to a measurement error 3 . The geologist choice is algorithm 3 with two votes. Although this algorithm detects less vugs than the others, this was preferred because of it provides better results (no false positive) in the dark strips.

Figure 3 shows the image input and output for each algorithm at depth2. In this case the input image shows few big vugs and algorithm 2 and 3 give best results, both gaining 2 votes. It is important to note that algorithm 3 shows, in general, a clear output and best accuracy, with a lower number of false positive. Detailed image results can be found in [12. The algorithm that produced the best overall results was the one based on the local threshold method. The second choice was the algorithm 2 . This indicates that, regardless the image shape, the convolution operator gives best results if it considers only actual image zones.

\section{Conclusions}

An approach for spot detection in images with noisy background has been proposed and tested in a real-world application. It consists of three main steps: image segmentation, image labeling and regions selection. We develop three different algorithms for image segmentation starting from well known techniques. The first and the second are based on convolution with a derivative circular mask and then a global thresholding technique is applied; the third algorithm uses a local threshold. To find actual objects we finally test some parameters (such as size and shape) of the identified regions. The approach was tested detecting vugs (spots in contrast with the noisy background) in a borehole image log. We evaluate our algorithms by a visual comparison of the obtained results, three domain experts then vote for the best algorithms. Results show that the algorithm 3, that uses a local threshold, was preferred by the domain expert. In general it detects less vugs than other algorithms, but it seems to be most suitable in all that cases with a low contrast between spots and background. Vugs detection is very important for the geologist who wants to evaluate the porosity of a rock, in order to quantify potential depth zones containing oil and gas. Our approach helps the geologist reducing the time for detection of vugs in the image logs and improving the detection accuracy. Outcomes from our algorithms can be considered as good starting points for porosity analysis, on which the geologist build his interpretation work.

Acknowledgements. This work has been partially supported by Camera di Commercio, Industria, Artigianato e Agricoltura di Ferrara, under the project "Image Processing and Artificial Vision for Image Classifications in Industrial Applications".

\footnotetext{
${ }^{3}$ This is an unavoidable error and can happens often in these type of image. Due to the complexity and the cost of the image acquisition, it is not possible to repeat the measurement. The final image is made by a single run over the entire well.
} 


\section{References}

1. Gonzalez, R.C., Woods, R.E.: Digital Image Processing, 3rd edn., pp. 689-794. Prentice-Hall, Englewood Cliffs (2008)

2. Shapiro, L.G., Stockman, G.C.: Computer Vision, pp. 279-325. Prentice-Hall, Englewood Cliffs (2001)

3. Datta, R., Joshi, D., Li, J., Wang, J.Z.: Image retrieval: Ideas, influences, and trends of the new age. ACM Comput. Surv. 40(2), Article 5 (2008)

4. Comaniciu, D., Meer, P.: Mean Shift: A Robust Approach toward Feature Space Analysis. IEEE Trans. Pattern Analysis Machine Intell. 24(5), 603-619 (2002)

5. Wang, J.Z., Li, J., Gray, R.M., Wiederhold, G.: Unsupervised Multiresolution Segmentation for Images with Low Depth of Field. IEEE Transactions on Pattern Analysis and Machine Intelligence 23(1), 85-90 (2001)

6. Tu, Z., Zhu, S.: Image Segmentation by Data-Driven Markov Chain Monte Carlo. IEEE Trans. on Pattern Analysis and Machine Intelligence 24(5) (May 2002)

7. Carson, C., Belongie, S., Greenspan, H., Malik, J.: Blobworld: Image segmentation using expectation-maximization and its application to image querying. IEEE Trans. Pattern Analysis and Machine Intelligence 24(8), 1026-1038 (2002)

8. Chen, J., Pappas, T., Mojsilovic, A., Rogowitz, B.: Adaptive image segmentation based on color and texture. In: Proceedings of the IEEE International Conference on Image Processing, ICIP (2002)

9. Deng, Y., Majunath, B.: Unsupervised segmentation of color-texture regions in images and video. IEEE Trans. Pattern Anal. Mach. Intell. 23(8), 800-810 (2001)

10. Otsu, N.: A threshold selection method from gray-level histograms. IEEE Trans. Systems, Man, and Cybernetics 9(1), 62-66 (1979)

11. Niblack, W.: An Introduction to Digital Image Processing. Prentice-Hall, Englewood Cliffs (1986)

12. Casarotti, L.: Algoritmi avanzati di analisi delle immagini da pozzi petroliferi (Advanced algorithm for borehole image processing). Master's Thesis, University of Ferrara (2011)

13. Ferraretti, D.: Analisi di immagini da pozzi petroliferi e loro classificazione (Borehole image analysis and classification). Master's Thesis, University of Ferrara (2006)

14. Abramoff, M.D., Magelhaes, P.J., Ram, S.J.: Image Processing with Image. J. Biophotonics International 11(7), 36-42 (2004)

15. Canny, J.: A computational approach to edge detection. IEEE Trans. Pattern Anal. Mach. Intell. 8(6), 679-698 (1986)

16. Serra, J.: Image Analysis and Mathematical Morphology. Academic Press, London (1982)

17. Mancas, M., Gosselin, B., Benoît, M.: Segmentation using a region-growing thresholding. Proceedings of the SPIE 5672, 12-13 (2005)

18. Ferraretti, D., Gamberoni, G., Lamma, E., Di Cuia, R., Turolla, C.: An AI Tool for the Petroleum Industry Based on Image Analysis and Hierarchical Clustering. In: Corchado, E., Yin, H. (eds.) IDEAL 2009. LNCS, vol. 5788, pp. 276-283. Springer, Heidelberg (2009) 